\title{
Sufficient conditions for oscillation of a nonlinear fractional nabla difference system
}

\author{
Wei Nian Li* and Weihong Sheng
}

\author{
*Correspondence: \\ wnli@263.net \\ Department of Mathematics, \\ Binzhou University, \\ Binzhou 256603, Shandong, \\ People's Republic of China
}

\section{Abstract}

In this paper, we study the oscillation of nonlinear fractional nabla difference equations of the form

$$
\left\{\begin{array}{l}
\nabla_{a}^{\alpha} x(t)+q(t) f(x(t))=g(t), \quad t \in \mathbb{N}_{a+1}, \\
\left.\nabla_{a}^{-(1-\alpha)} x(t)\right|_{t=a}=c,
\end{array}\right.
$$

where $c$ and $a$ are constants, $0<\alpha<1, \nabla_{a}^{\alpha}$ is the Riemann-Liouville fractional nabla difference operator of order $\alpha, a \geq 0$ is a real number, and $\mathbb{N}_{a+1}=\{a+1, a+2, \ldots\}$. Some sufficient conditions for oscillation are established.

Keywords: Oscillation, Nonlinear fractional nabla difference equation, Discrete fractional calculus

Mathematics Subject Classification: 26A33, 39A12, 39A21

\section{Background}

It is well known that discrete fractional calculus is a very new area. In 1988, Miller and Ross (1989) firstly introduced the definitions of non-integer order differences and sums. Since then, several authors started to study the theory of fractional difference equations. Especially, some excellent results have been established in recent years. For example, we refer the reader to Atici and Eloe (2007, 2009a, 2009b, 2011, 2012), Abdeljawad and Atici (2012), Atici and Wu (2014), Goodrich (2011, 2012), Anastassiou (2010, 2011), Čermák et al. (2015), Dassios and Baleanu (2013, 2015), Dassios et al. (2014), Hein et al. (2011), Abdeljawad (2011, 2013a, b), Alzabut and Abdeljawad (2014), Kang et al. (2014), Diblík (2015), Kisalar et al. (2015), Erbe et al. (2016), Li (2016) and the references therein.

The oscillation theory is an important part of the qualitative theory of fractional difference equations. However, to the best of our knowledge, few papers (Alzabut and Abdeljawad 2014; Kisalar et al. 2015; Li 2016) are known regarding the oscillatory behavior of fractional difference equations.

In Alzabut and Abdeljawad (2014), Alzabut and Abdeljawad considered the oscillation for nonlinear fractional difference equation of the form

$$
\begin{cases}\nabla_{a(q)-1}^{q} x(t)+f_{1}(t, x(t))=r(t)+f_{2}(t, x(t)), & t \in \mathbb{N}_{a(q)}, \\ \left.\nabla_{a(q)-1}^{-(1-q)} x(t)\right|_{t=a(q)}=x(a(q))=c, & c \in \mathbb{R},\end{cases}
$$


where $\mathbb{N}_{a(q)}=\{a(q)+1, a(q)+2, \ldots\}, a(q)=a+m-1, m=[q]+1, m-1<q<m$, $m \in \mathbb{N}, r: \mathbb{N}_{a(q)} \rightarrow \mathbb{R}, f_{i}: \mathbb{N}_{a(q)} \times \mathbb{R} \rightarrow \mathbb{R},(i=1,2), \nabla_{a(q)}^{q}$ is the Riemann-Liouville fractional nabla difference operator of order $q$ of $x$, and $\nabla_{a(q)}^{-q}$ is $q$-th fractional sum operator.

In Kisalar et al. (2015), Kisalar et al. studied oscillatory behavior of the higher order fractional nonlinear difference equations of the form

$\begin{cases}\Delta^{\alpha} x(t)+f_{1}(t, x(t+\alpha))=v(t)+f_{2}(t, x(t+\alpha)), & t \in \mathbb{N}_{0}, m-1<\alpha \leq m, \\ \left.\Delta^{\alpha-k} x(t)\right|_{t=0}=x_{k}, & k=1,2, \ldots, m-1,\end{cases}$

where $\Delta^{\alpha}$ is a Riemann-Liouville fractional delta difference operator of order $\alpha$, $m-1<\alpha \leq m, m \geq 1$ is an integer, $f_{i}:[0, \infty) \times \mathbb{R} \rightarrow \mathbb{R},(i=1,2)$ and $v$ are continuous with respect to $t$ and $x, \mathbb{N}_{a}=\{a, a+1, a+2, \ldots\}$.

In $\mathrm{Li}$ (2016), Li established the oscillation of forced fractional difference equations with damping term of the form

$$
(1+p(t)) \Delta\left(\Delta^{\alpha} x(t)\right)+p(t) \Delta^{\alpha} x(t)+f(t, x(t))=g(t), \quad t \in \mathbb{N}_{0},
$$

with initial condition $\left.\Delta^{\alpha-1} x(t)\right|_{t=0}=x_{0}$, where $0<\alpha<1$ is a constant, $\Delta^{\alpha} x$ is the Riemann-Liouville fractional delta difference operator of order $\alpha$ of $x$, and $\mathbb{N}_{0}=\{0,1,2, \ldots\}$.

In Atici and Eloe (2012), Atici and Eloe considered the following initial value problem for a nonlinear fractional difference equation

$\left\{\begin{array}{l}\nabla_{a}^{v} x(t)=f(t, x(t)), \quad \text { for } t=a+1, a+2, \ldots \\ \left.\nabla_{a}^{-(1-v)} x(t)\right|_{t=a}=x(a)=c,\end{array}\right.$

where $0<v \leq 1$ and $a$ is any real number. The authors obtained that $x(t)$ is a solution of (E4) if and only if

$$
x(t)=\frac{(t-a+1)^{\overline{v-1}}}{\Gamma(v)} x(a)+\nabla_{a+1}^{-v} f(t, x(t))
$$

Motivated by the papers (Atici and Eloe 2012; Alzabut and Abdeljawad 2014; Kisalar et al. 2015; Li 2016), in this paper, we investigate the oscillation of a nonlinear fractional nabla difference system of the form

$$
\left\{\begin{array}{l}
\nabla_{a}^{\alpha} x(t)+q(t) f(x(t))=g(t), \quad t \in \mathbb{N}_{a+1}, \\
\left.\nabla_{a}^{-(1-\alpha)} x(t)\right|_{t=a}=c
\end{array}\right.
$$

where $c$ and $\alpha$ are constants, $0<\alpha<1, \nabla_{a}^{\alpha}$ is the Riemann-Liouville fractional nabla difference operator of order $\alpha, a \geq 0$ is a real number, and $\mathbb{N}_{a+1}=\{a+1, a+2, \ldots\}$.

In this paper, we always assume that

(A) $f: \mathbb{R} \rightarrow \mathbb{R}$, and $x f(x)>0$ for $x \neq 0, g: \mathbb{N}_{a+1} \rightarrow \mathbb{R}$, and $q(t) \geq 0, t \in \mathbb{N}_{a+1}$.

A solution $x(t)$ of the system (1) is said to be oscillatory if it is neither eventually positive nor eventually negative, otherwise it is nonoscillatory. 


\section{Preliminaries}

In this section, we collect some basic definitions and lemmas that will be important to us in what follows. For an excellent introduction to the discrete fractional calculus, we refer the reader to papers (Atici and Eloe 2009a, 2012; Abdeljawad and Atici 2012; Anastassiou 2010, 2011; Abdeljawad 2011, 2013b).

Definition 1 (Atici and Eloe 2012) Let $v>0$. The $v$-th fractional $\operatorname{sum} f$ is defined by

$$
\nabla_{a}^{-v} f(t)=\frac{1}{\Gamma(v)} \sum_{s=a}^{t}(t-s+1)^{\overline{v-1}} f(s),
$$

for $t \in \mathbb{N}_{a}=\{a, a+1, a+2, \ldots\}$, where $\Gamma$ is the gamma function, and

$$
t^{\bar{\nu}}=\frac{\Gamma(t+v)}{\Gamma(t)} .
$$

Definition 2 (Atici and Eloe 2012) Let $\mu>0$ and $m-1<\mu<m$, where $m$ denotes a positive integer. Set $v=m-\mu$. The $\mu$-th fractional nabla difference is defined as

$$
\nabla_{a}^{\mu} f(t)=\nabla_{a}^{m-v} f(t)=\nabla_{a}^{m} \nabla_{a}^{-v} f(t) .
$$

Lemma 3 (Atici and Eloe 2012) Let $f$ be a real-valued function defined on $\mathbb{N}_{a}$, and let $\mu, v>0$. Then

$$
\nabla_{a}^{-v}\left[\nabla_{a}^{-\mu} f(t)\right]=\nabla_{a}^{-(\mu+v)} f(t)=\nabla_{a}^{-\mu}\left[\nabla_{a}^{-v} f(t)\right],
$$

and

$$
\nabla_{a+1}^{-v} \nabla f(t)=\nabla \nabla_{a}^{-v} f(t)-\frac{(t-a+1)^{\overline{v-1}}}{\Gamma(v)} f(a)
$$

Lemma 4 (Atici and Eloe 2012) For every $t \in \mathbb{N}_{a}$,

$$
\nabla_{a}^{-v}(t-a+1)^{\bar{\mu}}=\frac{\Gamma(\mu+1)}{\Gamma(\mu+v+1)}(t-a+1)^{\overline{v+\mu}} .
$$

Lemma 5 Let

$$
E(t)=\sum_{s=a}^{t}(t-s+1)^{\overline{-\alpha}} x(s), \quad t \in \mathbb{N}_{a} .
$$

Then

$$
\nabla E(t)=\Gamma(1-\alpha) \nabla_{a}^{\alpha} x(t) .
$$


Proof Using Definition 1, it follows from (8) that

$$
\begin{aligned}
E(t) & =\sum_{s=a}^{t}(t-s+1)^{\overline{-\alpha}} x(s)=\sum_{s=a}^{t}(t-s+1)^{\overline{(1-\alpha)-1}} x(s) \\
& =\Gamma(1-\alpha) \nabla_{a}^{-(1-\alpha)} x(t) .
\end{aligned}
$$

Using Definition 2, it follows from (10) that

$$
\nabla E(t)=\Gamma(1-\alpha) \nabla \nabla_{a}^{-(1-\alpha)} x(t)=\Gamma(1-\alpha) \nabla_{a}^{\alpha} x(t) .
$$

The proof of Lemma 5 is complete.

Lemma 6 Let $a \geq 0$ and $0<\alpha<1$ be real number, $u, v: \mathbb{N}_{a} \rightarrow \mathbb{R}$. If

$$
u(t) \leq v(t), \quad t \in \mathbb{N}_{a},
$$

then

$$
\nabla_{a}^{-\alpha} u(t) \leq \nabla_{a}^{-\alpha} v(t)
$$

Proof It follows from (11) that

$$
(t-s+1)^{\overline{\alpha-1}} u(s) \leq(t-s+1)^{\overline{\alpha-1}} v(s) \quad \text { for } s=a, a+1, \ldots, t
$$

Summing both sides of (13) from $a$ to $t$, we have

$$
\sum_{s=a}^{t}(t-s+1)^{\overline{\alpha-1}} u(s) \leq \sum_{s=a}^{t}(t-s+1)^{\overline{\alpha-1}} v(s) .
$$

Using Definition 1, from (14) we easily obtain (12). This completes the proof of Lemma 6.

\section{Main results}

In this section, we establish the oscillation results of system (1).

\section{Theorem 7 Assume that}

$$
\liminf _{t \rightarrow \infty}\left\{(t-a)^{1-\alpha} \sum_{s=a+1}^{t}(t-s+1)^{\overline{\alpha-1}} g(s)\right\}=-\infty
$$

and

$$
\limsup _{t \rightarrow \infty}\left\{(t-a)^{1-\alpha} \sum_{s=a+1}^{t}(t-s+1)^{\overline{\alpha-1}} g(s)\right\}=+\infty .
$$

Then every solution $x(t)$ of the system (1) is oscillatory. 
Proof Suppose to the contrary that there is a nonoscillatory solution $x(t)$ of system (1). It is obvious that there exists $t_{0} \in \mathbb{N}_{a+1}$ such that $x(t)>0$ or $x(t)<0, t \geq t_{0}$.

Case $1 x(t)>0, t \geq t_{0}$. Noting the assumption (A), from the system (1), we have

$$
\nabla_{a}^{\alpha} x(t)=-q(t) f(x(t))+g(t) \leq g(t) .
$$

Using Lemma 6, from (17), we have

$$
\nabla_{a+1}^{-\alpha} \nabla_{a}^{\alpha} x(t) \leq \nabla_{a+1}^{-\alpha} g(t) .
$$

Using Definition 2, Lemma 3 in the left-hand side of (18) and noting the initial condition of system (1) , we obtain

$$
\begin{aligned}
\nabla_{a+1}^{-\alpha} \nabla_{a}^{\alpha} x(t) & =\nabla_{a+1}^{-\alpha} \nabla \nabla_{a}^{-(1-\alpha)} x(t) \\
& =\nabla \nabla_{a}^{-\alpha} \nabla_{a}^{-(1-\alpha)} x(t)-\frac{(t-a+1)^{\overline{\alpha-1}}}{\Gamma(\alpha)} \nabla_{a}^{-(1-\alpha)} x(a) \\
& =x(t)-\frac{c}{\Gamma(\alpha)}(t-a+1)^{\overline{\alpha-1}} .
\end{aligned}
$$

Using Definition 1, it follows from the right-hand side of (18) that

$$
\nabla_{a+1}^{-\alpha} g(t)=\frac{1}{\Gamma(\alpha)} \sum_{s=a+1}^{t}(t-s+1)^{\overline{\alpha-1}} g(s) .
$$

Combining (18)-(20), we have

$$
x(t) \leq \frac{c}{\Gamma(\alpha)}(t-a+1)^{\overline{\alpha-1}}+\frac{1}{\Gamma(\alpha)} \sum_{s=a+1}^{t}(t-s+1)^{\overline{\alpha-1}} g(s) .
$$

It follows from (21) that

$$
\begin{aligned}
\Gamma(\alpha)(t-a)^{1-\alpha} x(t) \leq & c(t-a+1)^{\overline{\alpha-1}}(t-a)^{1-\alpha} \\
& +(t-a)^{1-\alpha} \sum_{s=a+1}^{t}(t-s+1)^{\overline{\alpha-1}} g(t) .
\end{aligned}
$$

By using the Stirling's formula (Alzabut and Abdeljawad 2014)

$$
\lim _{t \rightarrow \infty} \frac{\Gamma(t) t^{\varepsilon}}{\Gamma(t+\varepsilon)}=1, \quad \varepsilon>0,
$$

we obtain

$$
\begin{aligned}
& \lim _{t \rightarrow \infty}(t-a)^{1-\alpha}(t-a+1)^{\overline{\alpha-1}} \\
& \quad=\lim _{t \rightarrow \infty}(t-a)^{1-\alpha} \frac{\Gamma(t-a+1+\alpha-1)}{\Gamma(t-a+1)} \\
& \quad=\lim _{t \rightarrow \infty}(t-a)^{1-\alpha} \frac{\Gamma(t-a+\alpha)}{(t-a) \Gamma(t-a)} \\
& \quad=\lim _{t \rightarrow \infty} \frac{\Gamma(t-a+\alpha)}{(t-a)^{\alpha} \Gamma(t-a)} \\
& \quad=1 .
\end{aligned}
$$


Noting (23) and taking $t \rightarrow \infty$ in (22), we have

$$
\liminf _{t \rightarrow \infty}\left\{(t-a)^{1-\alpha} x(t)\right\} \leq-\infty
$$

which contradicts with $x(t)>0$.

Case $2 x(t)<0, t \geq t_{0}$. Noting the assumption (A), from the system (1), we have

$$
\nabla_{a}^{\alpha} x(t)=-q(t) f(x(t))+g(t) \geq g(t) .
$$

By Lemma 6, from (24), we obtain

$$
\nabla_{a+1}^{-\alpha} \nabla_{a}^{\alpha} x(t) \geq \nabla_{a+1}^{-\alpha} g(t)
$$

Using the procedure of Case 1, it follows from (25) that

$$
\begin{aligned}
\Gamma(\alpha)(t-a)^{1-\alpha} x(t) \geq & c(t-a+1)^{\overline{\alpha-1}}(t-a)^{1-\alpha} \\
& +(t-a)^{1-\alpha} \sum_{s=a+1}^{t}(t-s+1)^{\overline{\alpha-1}} g(t) .
\end{aligned}
$$

Noting (23) and taking $t \rightarrow \infty$ in (26), we have

$$
\limsup _{t \rightarrow \infty}\left\{(t-a)^{1-\alpha} x(t)\right\} \geq+\infty,
$$

which contradicts with $x(t)<0$. This completes the proof of Theorem 7 .

Theorem 8 Assume that there exists $t_{0} \in \mathbb{N}_{a+1}$ such that

$$
\liminf _{t \rightarrow \infty}\left\{\sum_{s=t_{0}+1}^{t} g(s)\right\}=-\infty
$$

and

$$
\limsup _{t \rightarrow \infty}\left\{\sum_{s=t_{0}+1}^{t} g(s)\right\}=+\infty .
$$

Then every solution $x(t)$ of the system (1) is oscillatory.

Proof Suppose to the contrary that there is a nonoscillatory solution $x(t)$ of system (1). It is obvious that there exists $t_{0} \in \mathbb{N}_{a+1}$ such that $x(t)>0$ or $x(t)<0, t \geq t_{0}$.

Case $1 x(t)>0, t \geq t_{0}$. As in the proof of Theorem 7, we obtain (17). Using Lemma 5, it follows from (17) that

$$
\nabla E(t) \leq \Gamma(1-\alpha) g(t)
$$


Summing both sides of (29) from $t_{0}+1$ to $t$, we have

$$
E(t) \leq E\left(t_{0}\right)+\Gamma(1-\alpha) \sum_{s=t_{0}+1}^{t} g(t)
$$

Letting $t \rightarrow \infty$ in (30), we obtain

$$
\liminf _{t \rightarrow \infty} E(t)=-\infty
$$

which contradicts with $E(t)>0$.

Case $2 x(t)<0, t \geq t_{0}$. As in the proof of Theorem 7, we obtain (24). Then, using the above mentioned method, we easily obtain a contradiction. This completes the proof of Theorem 8 .

\section{Remarks}

In our Definition 1, the fractional sum in (2) starts at $a$. In Abdeljawad and Atici (2012), Abdeljawad and Atici introduced the following fractional sum.

Let $v>0$. The $v$-th fractional $\operatorname{sum} f$ is defined by

$$
\widetilde{\nabla}_{a}^{-v} f(t)=\frac{1}{\Gamma(v)} \sum_{s=a+1}^{t}(t-s+1)^{\overline{v-1}} f(s), \quad t \in \mathbb{N}_{a+1} .
$$

Obviously, the fractional sum in (31) starts at $a+1$. In Abdeljawad and Atici (2012), the authors established the relation between the operators $\nabla_{a}^{-v}$ and $\widetilde{\nabla}_{a}^{-v}$ and considered the following initial value problem for a nonlinear fractional difference equation

$$
\left\{\begin{array}{l}
\nabla_{a-1}^{\alpha} x(t)=f(t, x(t)), \\
\left.\widetilde{\nabla}_{a-1}^{-(1-\alpha)} x(t)\right|_{t=a}=x(a)=c
\end{array} \quad \text { for } t=a+1, a+2, \ldots\right.
$$

where $0<\alpha<1$ and $a$ is any real number. The authors obtained that $x(t)$ is a solution of (E5) if and only if

$$
x(t)=\frac{(t-a+1)^{\overline{\alpha-1}}}{\Gamma(\alpha)} x(a)+\widetilde{\nabla}_{a}^{-\alpha} f(t, x(t)) .
$$

Using the idea in Abdeljawad and Atici (2012), we can try to investigate the oscillation of the following nonlinear fractional nabla difference system

$$
\left\{\begin{array}{l}
\nabla_{a-1}^{\alpha} x(t)+q(t) f(x(t))=g(t), \quad t \in \mathbb{N}_{a} \\
\left.\widetilde{\nabla}_{a-1}^{-(1-\alpha)} x(t)\right|_{t=a}=c
\end{array}\right.
$$

\section{Examples}

In this section, we give some examples to illustrate our main results. 
Example 9 Consider the following fractional nabla difference system

$$
\left\{\begin{array}{l}
\nabla_{1}^{\frac{1}{2}} x(t)+\frac{\left(t-\frac{\sqrt{\pi}}{2}\right) \Gamma(t)}{\Gamma\left(t+\frac{1}{2}\right)} x(t)=t, \quad t \in \mathbb{N}_{2}, \\
\left.\nabla_{1}^{-\frac{1}{2}} x(t)\right|_{t=1}=\frac{\sqrt{\pi}}{2}
\end{array}\right.
$$

Here $a=1, \alpha=\frac{1}{2}, q(t)=\frac{\left(t-\frac{\sqrt{\pi}}{2}\right) \Gamma(t)}{\Gamma\left(t+\frac{1}{2}\right)}, f(x(t))=x(t), g(t)=t$. It is easy to see that

$$
(t-1)^{\frac{1}{2}} \sum_{s=2}^{t}(t-s+1)^{\overline{-\frac{1}{2}}} g(s)=(t-1)^{\frac{1}{2}} \sum_{s=2}^{t}(t-s+1)^{\overline{-\frac{1}{2}}} s>0, \quad t \in \mathbb{N}_{2} .
$$

Therefore, from (33), we obtain that the condition (15) of Theorem 7 does not hold. Indeed, using Lemma 4 and Definition 1 , by careful calculation, we find that $x(t)=t^{\frac{1}{2}}>0$ is a nonoscillatory solution of system (32).

Example 10 Consider the following fractional nabla difference system

$$
\left\{\begin{array}{l}
\nabla_{1}^{\frac{1}{3}} x(t)+\frac{t^{2} \Gamma(t)}{\Gamma\left(t+\frac{1}{3}\right)} x(t)=t^{2}+\frac{1}{3} \Gamma\left(\frac{1}{3}\right), \quad t \in \mathbb{N}_{2} \\
\left.\nabla_{1}^{-\frac{2}{3}} x(t)\right|_{t=1}=\frac{1}{3} \Gamma\left(\frac{1}{3}\right)
\end{array}\right.
$$

Here $a=1, \alpha=\frac{1}{3}, q(t)=\frac{t^{2} \Gamma(t)}{\Gamma\left(t+\frac{1}{3}\right)}, f(x(t))=x(t), g(t)=t^{2}+\frac{1}{3} \Gamma\left(\frac{1}{3}\right)$. Obviously, for $t_{0} \in \mathbb{N}_{2}$,

$$
\sum_{s=t_{0}+1}^{t} g(s)=\sum_{s=t_{0}+1}^{t}\left(s^{2}+\frac{1}{3} \Gamma\left(\frac{1}{3}\right)\right)>0,
$$

which shows that the condition (27) of Theorem 8 does not hold. In fact, we can verify that $x(t)=t^{\frac{1}{3}}>0$ is a nonoscillatory solution of system (34).

Example 11 Consider the following fractional nabla difference system

$$
\begin{cases}\nabla_{1}^{\frac{1}{2}} x(t)+\frac{3 t}{\Gamma\left(t+\frac{1}{4}\right)} x(t)=(-1)^{t} e^{t}-(-1)^{t-1} e^{t-1}, & t \in \mathbb{N}_{2} \\ \left.\nabla_{1}^{-\frac{1}{2}} x(t)\right|_{t=1}=c_{1}, & \left(c_{1} \text { is a constant }\right) .\end{cases}
$$

Here $a=1, \alpha=\frac{1}{2}, q(t)=\frac{3 t}{\Gamma\left(t+\frac{1}{4}\right)} f(x(t))=x(t), g(t)=(-1)^{t} e^{t}-(-1)^{t-1} e^{t-1}$. We easily see that

$$
\sum_{s=t_{0}+1}^{t} g(s)=(-1)^{t} e^{t}-(-1)^{t_{0}} e^{t_{0}}, \quad t_{0} \in \mathbb{N}_{2}
$$


Therefore,

$$
\liminf _{t \rightarrow \infty}\left\{\sum_{s=t_{0}+1}^{t} g(s)\right\}=\liminf _{t \rightarrow \infty}\left\{(-1)^{t} e^{t}-(-1)^{t_{0}} e^{t_{0}}\right\}=-\infty
$$

and

$$
\limsup _{t \rightarrow \infty}\left\{\sum_{s=t_{0}+1}^{t} g(s)\right\}=\limsup _{t \rightarrow \infty}\left\{(-1)^{t} e^{t}-(-1)^{t_{0}} e^{t_{0}}\right\}=+\infty
$$

which show that the conditions in Theorem 8 are satisfied. By Theorem 8 , every solution $x(t)$ of the system (36) is oscillatory.

\section{Conclusions}

This paper provides some oscillation criteria for solutions of a nonlinear fractional nabla difference system by using the basic theories of discrete fractional calculus. The main results are given in Theorems 7 and 8 . In the end of this paper, we give three examples. Examples 9 and 10 show that the assumptions of the main results can not be dropped.

Authors' contributions

WNL and WS contributed equally to the writing of this paper. Both authors read and approved the final manuscript.

\section{Acknowlegements}

This work is supported by the National Natural Science Foundation of China (10971018). The authors thank the referees very much for their valuable comments and suggestions on this paper.

\section{Competing interests}

The authors declare that they have no competing interests.

Received: 8 April 2016 Accepted: 12 July 2016

Published online: 26 July 2016

\section{References}

Abdeljawad T (2013a) Dual identities in fractional difference calculus within Riemann. Adv Differ Equ. 2013:36. doi:10.1186/1687-1847-2013-36

Abdeljawad T (2013b) On delta and nabla Caputo fractional differences and dual identities. Discrete Dyn Nat Soc. 2013:406910. doi:10.1155/2013/406910

Abdeljawad T (2011) On Riemann and Caputo fractional differences. Comput Math Appl 62:1602-1611

Abdeljawad T, Atici FM (2012) On the definitions of nabla fractional differences. Abstr Appl Anal. 2012:406757. doi:10.1155/2012/406757

Alzabut JO, Abdeljawad T (2014) Sufficient conditions for the oscillation of nonlinear fractional difference equations. J Fract Calc Appl 5:177-187

Anastassiou GA (2010) Nabla discrete fractional calculus and nabla inequalities. Math Comput Model 51:562-571 Anastassiou GA (2011) Right nabla discrete fractional calculus. Int J Differ Equ 6(2):91-104

Atici FM, Eloe PW (2007) A transform method in discrete fractional calculus. Int J Differ Equ 2(2):165-176

Atici FM, Eloe PW (2009) Discrete fractional calculus with the nabla operator. Electron J Qual Theory Differ Equ Spec Ed I $3: 1-12$

Atici FM, Eloe PW (2009) Initial value problems in discrete fractional calculus. Proc Am Math Soc 137:981-989 Atici FM, Eloe PW (2011) Linear systems of fractional nabla difference equations. Rocky mountain. J Math 41:353-370

Atici FM, Eloe PW (2012) Gronwall's inequality on discrete fractional calculus. Comput Math Appl 64:3193-3200

Atici FM, Wu F (2014) Existence of solutions for nonlinear fractional difference equations with initial conditions. Dyn Syst Appl 23:265-276

Čermák J, Gyori l, Nechvátal L (2015) On explicit stability conditions for a linear fractional difference system. Fract Calc Appl Anal 18:651-672

Dassios IK, Baleanu DI (2013) On a singular system of fractional nabla difference equations with boundary conditions. Bound Value Probl. 2013:148. doi:10.1186/1687-2770-2013-148

Dassios IK, Baleanu DI, Kalogeropoulos GI (2014) On non-homogeneous singular systems of fractional nabla difference equations. Appl Math Comput 227:112-131 
Dassios IK, Baleanu DI (2015) Duality of singular linear systems of fractional nabla difference equations. Appl Math Model 39:4180-4195

Diblík J, Fečkan M, Pospišil M (2015) Nonexistence of periodic solutions and S-asymptotically periodic solutions in fractional difference equations. Appl Math Comput 257:230-240

Erbe L, Goodrich CS, Jia B, Peterson A (2016) Survey of the qualitative properties of fractional difference operators: monotonicity, convexity, and asymptotic behavior of solutions. Adv Differ Equ. 2016:43. doi:10.1186/s13662-016-0760-3

Goodrich CS (2011) Existence of a positive solution to a system of discrete fractional boundary value problems. Appl Math Comput 217:4740-4753

Goodrich CS (2012) On a discrete fractional three-point boundary value problem. J Differ Equ Appl 18:397-415

Hein J, McCarthy Z, Gaswick N, McKain B, Hill K (2011) Laplace transforms for the nabla-difference operator. Panam Math J 21(3):79-97

Kang S, Li Y, Chen H (2014) Positive solutions to boundary value problems of fractional difference equation with nonlocal conditions. Adv Differ Equ. 2014:7. doi:10.1186/1687-1847-2014-7

Kisalar S, Yildiz MK, Aktoprak E (2015) Oscillation of higher order fractional nonlinear difference equations. Int J Differ Equ 10(2):201-212

LiWN (2016) Oscillation results for certain forced fractional difference equations with damping term. Adv Differ Equ. 2016:70. doi:10.1186/s13662-016-0798-2

Miller KS, Ross B (1989) Fractional difference calculus. In: Proceedings of the international symposium on univalent functions, fractional calculus and their applications. Nihon University, Koriyama, Japan, May 1988, pp 139-152. Ellis Horwood Ser Math Appl, Horwood, Chichester

\section{Submit your manuscript to a SpringerOpen ${ }^{\circ}$ journal and benefit from:}

- Convenient online submission

\section{- Rigorous peer review}

- Immediate publication on acceptance

Open access: articles freely available online

- High visibility within the field

Retaining the copyright to your article

Submit your next manuscript at $>$ springeropen.com 